\title{
A Plan-based Dialogue Model Using a Discourse Stack for Intelligent Human- Robot Interface Development
}

\author{
Sangwoo Kang \\ Dep. of Computer Science \\ Sogang University, Korea \\ swkang@sogang.ac.kr
}

\author{
Youngjoong Ko \\ Dep. of Computer Engineering \\ Dong-A University, Korea \\ yjko@dau.ac.kr
}

\author{
Jungyun Seo \\ Dep. of Computer Science \\ Sogang University, Korea \\ seojy@sogang.ac.kr
}

\begin{abstract}
The existing studies of a dialogue system can be classified into two major parts. One is a study for a practical system, and the other is a study to understand a principal of dialogue phenomena. The former focuses on robustness in practical environment for dialogue systems. However, it cannot guarantee its performance in complicated dialogue environment. The latter has studied as the plan-based model typically. It has some strong points that it can reflect complex dialogue phenomena and can infer user's intention in various situations. However, since an initial design of this model is so complicated, it is difficult for this model to be extended to effective response generation in a practical dialogue system.

This paper proposes a new dialogue model using a simplified plan recognition technique and a discourse stack to effectively generate responses in a practical domain.
\end{abstract}

\section{Introduction}

The dialogue to the human is the most natural and effective communication method to achieve the desired task. The studies to use a dialogue as the communication means of the human and machines have been in progress and the several practical dialogue systems recently are available in the practical environment. The dialogue system is software which provides the human an interaction method with machines through the natural language and it is superior to the other interaction methods due to excellent flexibility and expressiveness.

Generally, the dialogue system carries out two roles of the task execution and information offering according to the object of dialogue. If a dialogue makes progress to perform the specific work, it is called by the task-oriented dialogue, and if it makes progress to gain information, it is the information seeking dialogue. The task-oriented dialogue and the information-seeking dialogue are mixed together in the human dialogues, and they have very complicated forms in their structure. To effectively handle these complicated human dialogues, we need to build up a well-designed dialogue system, which has to contain the intellectual work performance, domain flexibility for various applications, and a robust structure for minimizing errors.

In the proposed model, the dialogue system which uses the discourse stack and a plan-based model are developed to satisfy the above conditions. The planbased model, which is simplified and uses a discourse stack, can efficiently process complicated discourse phenomena and generate effective responses.

Generally, the dialogue system answers the direct request of a user. However, when a user wants to do any complicated task which is composed of many tasks, he/she can meet many problems or errors. The proposed model includes a process which infers the ultimate request of a user using plan inference engine and then verify using dialogue stack.

\section{Related work}

The previous studies about the dialogue system are divided into two research fields. The first research is for the systems which can guarantee the robust performance in the practical environment. The range of this research is limited to simple and restrictive domains and there is no variation of the dialogue or predictable dialogue sequence type. The one of model for the first research field is the script-based model. The second research focuses on the dialogue model to understand complicated and various dialogue pheno- 
mena. The typical model for the second field is the plan-based model.

Recently, the researchers have introduced methods based-on probability-based models and they can solve the problems at the error propagation of each intermodule.

\subsection{A script based dialogue model}

A script-based dialogue model mostly used the DTN (Dialogue Transition Network). This model is very useful to process the job procedure for performing the specific purpose successfully. And this model is generally used in the format-based dialogue model (form-based dialogue model)[1].

The DTN model expresses the dialogue structure of the user utterance which implements the state and allowed transition of a dialogue in the network. A user can maintain only the state where he defined in advance and the movement to next state is made through the transition existed in a network. However, this model can be robustly operated and it can be simply designed. Therefore, this model is appropriate in the domains which maintain to exclude an ambiguity and to require robustness. However, because this model uses the sequential structure, it is hard to guarantee its high portability and flexibility.

\subsection{A plan based dialogue model}

The basic concept of the plan-based model is to infer the intention which user wants from the observed elementary action(plan recognition). This model can process various inferences according to the state of the user belief from the same condition, and this model can explain a complicated dialogue structure using formal domain knowledge(recipe). Kautz, Litman and Allen[2, 3] applied the plan theory to a dialogue system filed for the first time. They divided the dialogue structure into the discourse level and domain level. Their model can identify user's deep speech-act using inference rules in a discourse level and recognize user's intention in a domain level by using user's belief. A dialogue structure is expressed as the plan tree. One user utterance can generate plan trees which are diverse according to its situation. Therefore, the plan-recognition is a task that guesses a most well set of the plans which can explain an observed behavior.

Lambert[4,5] added the problem-solving level between discourse level and domain level in order to handle the more complicated dialogue structure. This level connects of domain level and its related discourse level. Therefore, the several user utterances could have a correlation and the related plan trees can be comprised of single tree.

Kautz, Litman, Allen and Lambert presented the model to explain the dialogue of the human-human dialogue. However, Eller[6] and Chu-Carrol[7,8] presented the model in which the recognition model and response generation model of a user are integrated. They presented the system's plan tree corresponding to the user's plan tree. Their model can independently maintain a user and a system state through two plan trees. Therefore, the state of the mutually different knowledge between the dialogue participants can be modeled. This system can carry out a dialogue which includes knowledge conflicts between the dialogue participants through the negotiation dialogue. The negotiation dialogue makes use of propose-evaluate-modify cycle. When knowledge conflicts between the dialogue participants are occurred, this cycle produces the system response through the process of changing the user belief by using the 'propose' of alternative and 'evaluation'.

Recently, the Allen[9] presented a system which is possibly learned by his plan based model. However, this system considers only the several predefined dialogues.

\subsection{A statistics based dialogue model}

Recently, the researchers attempt to combine with the speech recognition system. Thus, they attempt to overcome the effectiveness of error propagation problems and maintenance through the probability based model.

Roy[10] presented a method that defines the set of the possible dialogue states and actions, and it predicts the transition probability between the states.

The Singh[11] presented a method to automatically improve the dialogue strategy of a system through the reward of a user using the reinforcement learning. However, only very small states can be defined in this method. Because the process of repetitively learning is needed, this model is not useful in practical environment.

\section{A Plan Based Dialogue Model for Effective Response Generation}

The dialogue system can be built for the task oriented dialogue and information acquisition dialogue in many domains, and it has to include intellectual dialogue strategies. Moreover, it must have the structure with easy maintenance and portability. The system which has intellectual dialogue strategies, even 


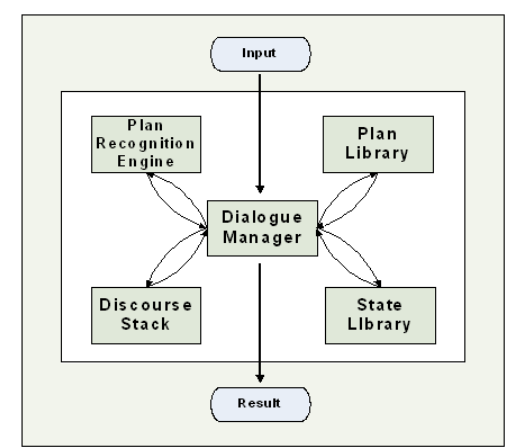

Figure 1 . The system architecture

if a user does not speak the explicit intention, can find the deep intention.

Actually, the task-oriented and information-seeking dialogues are mixed in many man-machine dialogues of practical applications. To apply it to our proposed system, our model uses the plan-based model to recognize the deep intention of a user. Also, we simplify the plan based model to have a robust structure for maintenance and management.

As shown in Figure 1, the plan recognition engine finds all possible plan trees from on the utterance of a user. The dialogue manager recognizes the intention of user by using dialogue strategy and selecting the most appropriated response at the current state. The plan library represents knowledge required for plan recognition and the state library stores the user state and world knowledge. The discourse stack stores the dialogue structure of user and system, and the dialog manager uses the discourse stack according to the dialogue strategy.

\subsection{Plan representation}

We modified forms of the plan library which Allen presented for representing knowledge of plan recognition(Figure 2). The attributes of the modified form are composed of the plan name, constraint condition, precondition, decomposition, effect and goal.

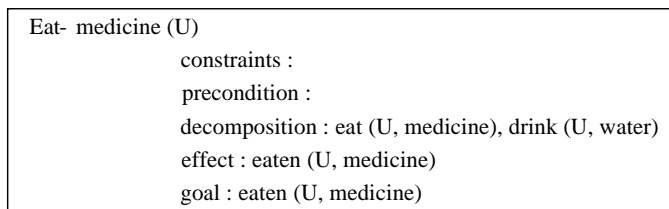

Figure 1. An example of a plan representation

The plan name is the unit of a plan, precondition includes conditions that it is already resolutely satisfied so that the corresponding plan can be selected. The constraint is conditions that have to solve in order to make plans which are currently selected. The current plan is satisfied only when lower plans in decomposition are altogether satisfied. An effect is the proposition in which it is certainly true when the current plan was satisfied. And a goal is the proposition in which it is expect to be true when the current plan was satisfied.

\subsection{A recognition model for user intention}

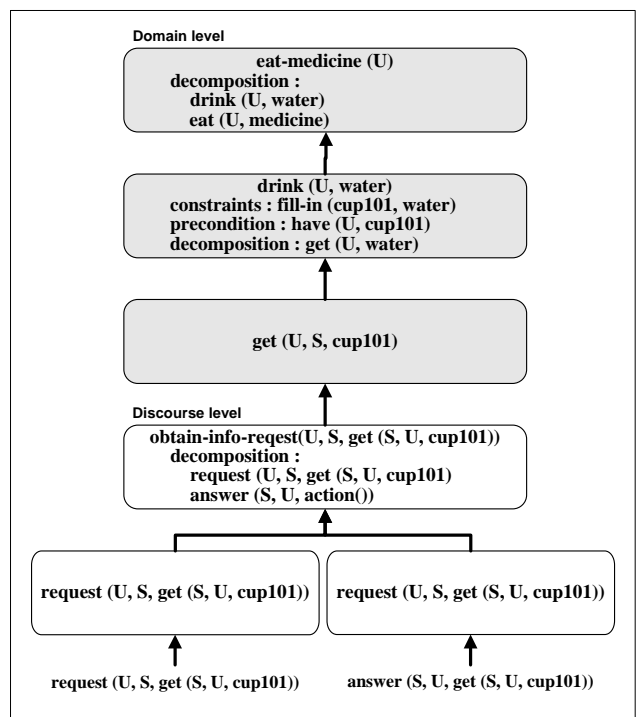

Figure 2. An example inferred-plan tree

Recognizing the dialogue object of a user in the dialogue system is very important for efficient dialogue process. We use two-stage plan recognition method for simple structure. In this system, the language analysis result of user utterance is used as the input of the plan recognition and then it is changed into a predicate representation format.

The first stage of the plan recognition is the process of discourse level. Since our discourse level represents discourse structure, it organizes a dialogue by using the principles of the adjacency pair[12,13]. For example, the adjacency pair is a dialogue structure like question and reply. According to the level of a dialogue, it can be hierarchically constructed. Moreover, structural plans(e.g. obtain-info-request()) for connecting adjacency pairs with the single plan are included in this discourse level.

The second stage of the plan recognition is the process of domain level. The domain level includes all plans expressed as the elementary action. In this stage, all plan trees that it can infer from the discourse 
level is produced. Then the dialog manager controls an inference level with reference to the state library.

Plan trees are constructed by inference rules. In the same level, if the plan name of a lower plan is included in the decomposition of a upper plan, these two plans can be connected. It is docomposition-subaction rule. There is a goal-precondition rule for between the other levels. If the goal for the plan of discourse level is consistent with the precondition of the plan of the domain level, these two plans can be connected (See Figure 3).

\subsection{Response generation strategies}

In the previous paragraph, we explain the process of getting an inference consequence about the user input by using the plan recognition and plan library.

The process for response generation is here explained. In Figure 3, the plan tree about the user input is divided into three levels. The initial inference level infers 'get(U, S, cup101)' from elementary action, and because a user is under the required behavior, this is true clearly. However, the deep intention of a user can be caught from the more intellectual dialogue system. Therefore, the dialog manager gets results that it infers 'drink(U, water)' or 'eat-medicine(U)' by using upper plan information from the plan inference engine. However, because these plan trees is only possible plan, we cannot decide a plan as user's intention. Therefore, the system decides the user's intention through the confirmation dialogue strategy. For efficient system response generation in our plan-based model, we propose discourse stack structure that manages to unify main-dialogue and subdialogue. A main-dialogue directly relates to user's intention and a sub-dialogue means the functional dialogue for task completion. Since a sub-dialog is a dialogue which requests the insufficient information, a system confirmation belongs to this sub-dialogue.

The discourse stack operation regulation for generating the system response is shown as follows:

\section{Discourse Stack Operation Rules}

Common Rule: If a top position item of DS is system response, the system generates system response using the item.

step 1 : to store surface user intention.

step 2 : if the plan recognition engine generates deep user intentions, a domain-confirm question related to those intentions is stored in DS.

i DM decides the priority of plan trees using the state library.

ii If the user response of the domain-confirm question is 'yes', plans related to the domain-confirm question is delete from DS.

iii If the user response of the domain-confirm question is 'no', the next domain-confirm question is selected. step 3 : if constraints are not satisfied at current plan, a constraint-confirm question related to the constraint is stored in DS.

Repeat processes for step 2 and step 3 until only the surface user intention stored in step1 is left in DS.

Figure 4 shows the process where the discourse stack is operated. When the dialog manager received "Get cup!” as an input, the system stored surface user's intention(e.g. get(U, S, cup101)) by a step 1 . Then the plan recognition engine infers the user's deep intentions. Since these intention are a case of bringing a cup in order to drink the water(e.g. $\operatorname{drink}(\mathrm{U}$, water)), this case is expected for user to take a medicine (e.g eat-medicine(U)). Therefore, in a step 2, in order to decide what a user wants during these plans, the system generates a confirmation question using the discourse stack. The order stored in the discourse stack is very important because dialogue is progressed according to the stored order. This order is determined from the result of the user modeling is stored in the state library. In Figure 4, if it currently is the time when a user has to take a medicine, dialogue manager stores a confirm-ation question in the upper part of a stack. If a user responds positively about the confirmation question(e.g. domain-confirm-question(S, $\mathrm{U}$, eat-medicine(U))), a confirmation question stored in a stack is deleted. On the other hand, if a user responds negatively, the dialogue manager selects the next confirmation question.

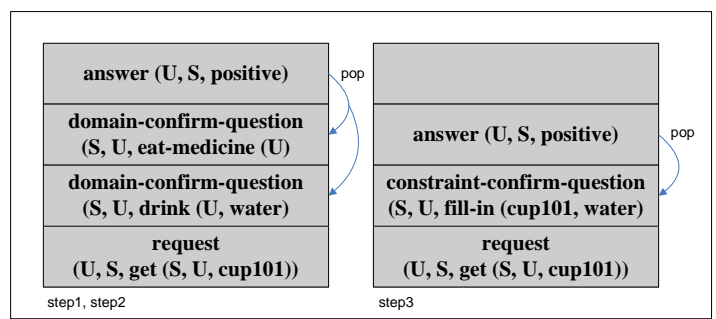

Figure 3. An example for the usage of discourse stack

In Step 3, if a constraint that is not satisfied in the plan is remained, the dialog manager generates a confirmation for resolving this condition by using discourse stack. In Figure 3, the constraint condition of the plan(e.g. drink(U,water)) includes constraint (e.g. fill-in(cup101, water)). If there is no water in a cup, a confirmation question(e.g "is the water filled in cup?") is put into the discourse stack(see Figure 4). Therefore, the constraint can be effectually handled. When conditions of all plans were satisfied by user's response, the dialogue manager empties a discourse stack and completes the task. 


\section{Comparison with previous approach}

The proposed model is a new modeling technique of the plan-based model to generate the effective response. However, a comparison with the other system is very difficult quantitatively. Therefore, in this section, we compare our proposed method to the previous method using an example. Example 1and 2 is the case where a user requests a request, "get cup", in the time to take a medicine. Example 1 is a general process of a previous system. Because the system cannot recognize user's deep intention, this system has to process all three tasks about a user requests. In this case, because a user has every time to order each task and a system has to recognize and process, a task completion rate is dramatically decreased.

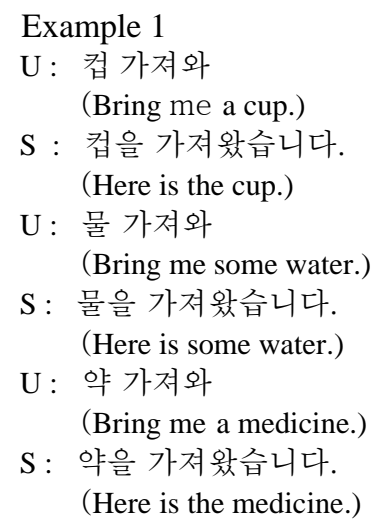

Example 2 is our system's initiative dialogue which is progressed by confirming the task. Therefore, a user can progress a dialogue about the question of a system with the simple response. As considering the speech recognition and task completion, the system can progress the very effective dialogue. In the aspect of dialogue contents, the proposed model can be flexible according to the condition state and it is possible to generate the more intellectual response.

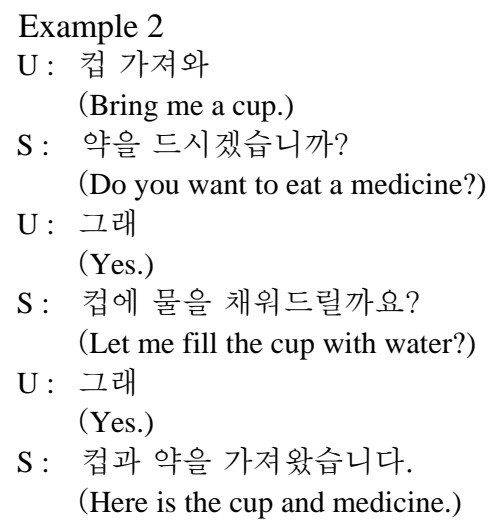

\section{Conclusion}

We propose a new dialogue model which has robust and efficient structures to resolve complicated discourse phenomena in practical environment. The proposed model is based on plan recognition and it efficiently generates system responses using a discourse stack for the effective confirmation and inference of the upper plan.

Also we explain an advantage of the proposed model using an example because a quantitative comparison of the dialogue system is very difficult. In future work, we will apply evaluating methods like simulated user and developing a dialogue model which is similar to human-dialogue.

\section{Acknowledgments}

This research (paper) was performed for the Intelligent Robotics Development Program, one of the 21st Century Frontier R\&D Programs funded by the Ministry of Commerce, Industry and Energy of Korea.

\section{References}

[1] David Goddeau, Helen Meng, Joe Polifroni, Stephanie Seneff, and Senis Busayapongchai, “A Form-Based Dialog Manager for Spoken Language Applications," Proceedings of the $4^{\text {th }}$ International Conference On Spoken Language Processing, pp701705, 1996.

[2] Henry Kautz and James Allen. "Generalized Plan Recognition", Proceedings of the $14^{\text {th }}$ National Conference on Artificial Intelligence, pp 32-37, 1986.

[3] Diane Litman and James Allen, “A Plan Recognition Model for Subdialogue in Dialogues”, Cognitive Science, vol 11, pp163-200, 1987.

[4] Lynn Lambert and Sandra Carberry, “A tripartite plan-based model of dialogue," Proceedings of the $29^{\text {th }}$ Annual Meeting of the Association for Computational Linguistics, pp47-54, 1991.

[5] Lynn Lambert, Recognizing Complex Discourse Acts : A Tripartite Plan- Based Model of Dialogue, Ph.D. thesis, University of Delaware, 1993.

[6] Rhonda Eller and Sandra Carberry, "A meta-rule approach to flexible plan recognition in dialogue," User Modeling and User-Adapted interaction, vol. 2, num 1-2, pp27-53, 1992.

[7] Jennifer Chu-Carroll and Sandra Carberry, "Generating information-sharing sub-dialogues in expert-user consultation," Proceedings of the $14^{\text {th }}$ International Conference on Artificial Intelligence, pp1234-1250, 1995. 
[8] Chu-Carroll and Carberry, "Conflict Resolution in Collaborative Planning Dialogues,” International Journal of Human-Computer Studies, vol. 53(6), pp. 969-1015, 2000.

[9] James Allen, Nathanael Chambers, George Ferguson, Lucian Galescu, Hyuckchul Jung, Mary Swift, and William Taysom, "PLOW: A Collaborative Task Learning Agent," Proceedings of the $21^{\text {th }}$ AAAI Conference on Artificial Intelligence, pp1514-1519, 2007

[10] Nicholas Roy, Joelle Pineau and Sebastian Thrun, "Spoken Dialog Management Probabilistic Reason- ing," Proceedings of the $38^{\text {th }}$ Annual Meeting of the Association for Computational Linguistics, pp. 93-100, 2000

[11] Satinder singh, Diane Litman, Michael Kearns and Marilyn Walker, "Optimizing Dialogue Management with reinforcement Learning," Journal of artificial Intelligence Research, vol. 16, pp.105-133, 2002

[12] E. Schegloff and H. Sacks, "Opening up closings,” Semiotica, vol. 7(4), pp. 289-327, 1973.

[13] Stephan Levinson, Pragmatics, Cambridge University Press, Cambridge, UK, 1983. 